\title{
sciendo
}

\section{Relationships Between Measures of Functional and Isometric Lower Body Strength, Aerobic Capacity, Anaerobic Power, Sprint and Countermovement Jump Performance in Professional Soccer Players}

\author{
by

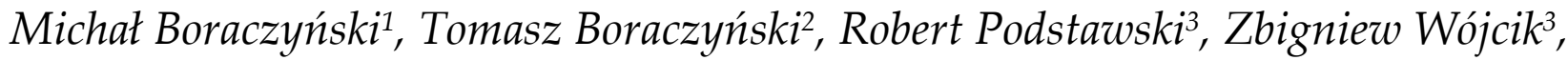 \\ Piotr Gronek ${ }^{4}$
}

\begin{abstract}
The purpose of this study was to assess a wide range of physiological and performance variables and investigate whether and to what extent these variables are associated with each other in soccer. Twenty-five male soccer players $(25.1 \pm 4.56$ years; body mass, $75.2 \pm 5.92 \mathrm{~kg}$; body height, $180.6 \pm 5.45 \mathrm{~cm})$ performed: 5 - and $30-\mathrm{m}$ sprints (T5m and T30m, respectively), 1-repetition-maximum (1RM) half squat, maximal voluntary isometric contraction (MVIC) of the knee extensors, countermovement jump (CMJ) to obtain vertical jump height (CMJheight) and power output (CMJpower), the 10-s Wingate Anaerobic Test (WAnT) to obtain peak power $\left(P_{\max }\right)$, and the 20-m multi-stage shuttle run test (MST) to evaluate aerobic capacity. 1RM, MVIC, and $P_{\max }$ were normalized to body mass. Large negative correlations were found between sprint times and 1RM half back squat/BM $\left(r=-0.510\right.$ to $-0.570, r^{2}=0.260-0.325$, both $\left.p<0.01\right)$ and $P_{\max } / B M\left(r=-0.501, r^{2}=0.251, p<0.01\right)$. T30m most strongly and negatively correlated with $C M \mathrm{~J}_{\text {height }}(r=-0.744$, $\left.r^{2}=0.554, p<0.001\right)$. WAnT-determined $P_{\max }$ showed a very large correlation between absolute $P_{\max }$ and knee-extensor MVIC $\left(r=0.827, r^{2}=0.684, p<0.001\right)$ and large correlations between absolute $P_{\max }$ and 1 RM half squat $\left(r=0.674, r^{2}\right.$ $=0.454, p<0.001)$ and $C M J_{\text {power }}\left(r=0.579, r^{2}=0.335, p<0.01\right)$. We also identified a large inverse relationship between $C M J_{\text {height }}$ and T30m $\left(r=-0.744, r^{2}=0.554, p<0.001\right)$ and large positive correlation between CMJheight and MVIC/BM $(r$ $=0.702, p<0.001$ ). The results demonstrate that elite soccer players with greater lower body strength (quantified by the MVIC of the knee extensor and the 1RM half squat) show better sprint and CMJ performance, suggesting the incorporation of soccer-specific resistance training to develop lower body musculature and therefore maximize sprinting ability. The higher correlation coefficients found between T30m and the physiological and athletic measures compared with T5m promote the use of this sprint distance when assessing performance. The use of relative measures (normalized to body mass) is advisable when comparing strength variables with sprint and CMJ performance or anaerobic power. Considering the correlations of WAnT-determined $P_{\max }$ versus CMJpover, coaches should administer tests that assess jumping and linear sprint performance rather than the cycling-specific WAnT.
\end{abstract}

Key words: static and dynamic muscular strength, sprinting ability, acceleration, physiological response, endurance shuttle run, competitive soccer.

\section{Introduction}

Competitive soccer is a team sport that involves acyclic and unpredictable movement patterns (sensory-motor sequences of actions), combining numerous short explosive bouts of activity and relatively few extended bouts of mild

\footnotetext{
1 - Department of Public Health, Collegium Medicum, University of Warmia and Mazury, Olsztyn, Poland.

2 - Department of Health Sciences, Olsztyn University, Olsztyn, Poland.

3 - Department of Tourism, Recreation and Ecology, University of Warmia and Mazury, Olsztyn, Poland.

4 - Department of Dance and Gymnastics, Poznan University of Physical Education, Poznań, Poland.
} 
to intense efforts (Bartlett et al., 2012; Little and Williams, 2007). During a game, players change activity on average every $5 \mathrm{~s}$ and perform approximately 1300 diverse activities (e.g., jogging, sprinting, turning, dribbling, kicking, tackling, and jumping) with nearly 200 of these being completed at high or near-maximal intensity that corresponds to $\geq 85 \%$ of maximal oxygen uptake $\left(\mathrm{VO}_{2 \max }\right)$ or $80-100 \%$ heart rate maximum (HRmax) (Bangsbo et al., 2006). In elite soccer, players must generally run at a high intensity every $60 \mathrm{~s}$ and sprint all-out once every 4 min (Strudwick and Reilly, 2001). These intermittent bouts of prolonged high-intensity exercise highlight the critical role of anaerobic metabolism in soccer performance (Dupont et al., 2004; Osgnach et al., 2010). However, high aerobic fitness is essential as aerobic metabolism accounts for $90 \%$ of the energy contribution during regular match play (Stolen et al., 2005). Several crosssectional studies have reported a significant relationship between aerobic fitness $\left(\mathrm{VO}_{2 \max }\right.$, lactate threshold, and running economy) and variables that quantify soccer performance such as competitive ranking, team level, and total distance covered during a match (Castagna et al., 2002; Krustup et al., 2003; Ziogas et al., 2011).

In addition to aerobic and anaerobic conditioning, previous research has identified speed, agility, strength, and power as important determinants of soccer performance (Hoff and Helgerud, 2004). While these physiological qualities do not need to be highly advanced compared with other athletes' populations, soccer players must develop and maintain a very high level of performance in order to be successful (Reilly et al., 2000). Although it is practical for strength and conditioning specialists and coaches to develop strategies that would simultaneously improve these components of fitness together with the necessary technical and tactical skills, the inter-individual relationships between these abilities and other physical, physiological, and metabolic attributes with the variables that quantify soccer performance are still unclear and inconsistent (Meckel et al., 2009; Wadley and Le Rossignol, 1998). A number of studies have found certain physiological qualities are strongly associated with game-related physical performance variables, finding large correlations between repeated sprint ability (RSA) and highintensity running and sprinting distances
(Rampinini et al., 2007), 1 repetition maximum (1RM) half back squat and 10-m sprint time (Wisloff et al., 2004), and relative strength (1RM/body mass) and short sprint performance (<30m) (Comfort et al., 2014; McBride et al., 2009). However, other studies have reported nonsignificant or very weak correlations such as between Smith machine squat performance and 10-m and 40-m sprint times (Harris et al., 2008) as well as between aerobic fitness $\left(\mathrm{VO}_{2 \max }\right)$ and RSA in team-sports athletes (Glaister, 2005; Tomlin and Wenger, 2001). In addition, soccer-specific research has found that agility, acceleration, and maximum speed are in fact unrelated components and that these qualities require independent training as well as testing methods (Little and Williams, 2005).

Given the conflicting evidence in the literature, additional correlative research is needed to better elucidate these relationships. Furthermore, a significant limitation of the aforementioned studies is that many involved amateur or semi-professional players with low to moderate training experience (Aguiar et al., 2008; Nassis et al., 2010). Research involving elite players is rare and thus warranted. Another issue is that many of these studies applied different testing modalities and protocols which hinders between-study comparisons (Glaister, 2005; Tomlin and Wenger, 2001). As a result, research based on comparative methodology to previous works would enhance the validity of present findings. Therefore, the aims of the present study were to (A) assess a wide range of physiological and athletic performance variables, and (B) investigate whether and to what extent these variables are associated with each other in professional soccer. It was assumed that those tasks and performance measures that involve a similar metabolic pathway and exercise-time relationship would produce the highest correlations.

\section{Methods}

\section{Participants}

Twenty-five male soccer players aged 25.1 \pm 4.56 years (mean $\pm \mathrm{SD}$ ) were recruited from the same first division team. The sample consisted of 8 defenders, 12 midfield players, and 5 forwards. No goalkeepers were involved in the study. All were full-time professional soccer players with 12-15 years of experience and trained $2-3 \mathrm{~h}$ per 
day $\left(14 \pm 2.6 \mathrm{~h} \times\right.$ week $\left.^{-1}\right)$ with a control match played every weekend. Participation was voluntary and written informed consent was provided after the purpose, potential risks, and procedures of the study were explained. Each participant was required to possess official medical clearance in accordance with the national law as an indicator of positive health status.

\section{Design and Procedures}

The study received the approval of the Ethics Committee for Studies Involving the Use of Human Subjects. All procedures were performed in accordance with the 2013 Declaration of Helsinki. A descriptive correlational design was employed to examine the associations between various physiological and performance test variables. Linear regression analysis was used to evaluate the relationships between each of these variables and the results were analyzed to determine the relationships. All testing was performed in March in the pre-season conditioning period in order to control for extrinsic factors and integrated with the preseason training regime of participants.

Testing was conducted on two separate days with a minimum 1-day and a maximum 3day interval between training sessions. Sprint performance, lower extremity dynamic and static strength, countermovement jump performance, and anaerobic power were assessed on the first day and aerobic performance with blood lactate measures was measured on the second day (in the described order). Players were instructed to avoid intense exercise $\left(>85 \% \mathrm{HR}_{\max }\right)$ in the 24-hour period before testing in order to minimize the effects of fatigue. All tests were performed before noon (10.00-11.30) and conducted in the same order for all players. Sufficient rest was provided between all trials and tests to allow for recovery. All of the participants were familiar with the administered tests due to frequent performance assessments (eight times in the previous 2 years) involving the same test protocols.

Testing on the first day was conducted in a laboratory $\left(20.1-21.8^{\circ}\right.$ C, $32-41 \%$ relative humidity, $758-764 \mathrm{mmHg}$ ) and on the second day in a gym $(19.7-20.9 \circ \quad$ C) to measure aerobic performance. On the first day of testing participants were evaluated for standing body height to the nearest $0.1 \mathrm{~mm}$ with a calibrated stadiometer (WB-150, Tryb-Wag ZPU, Zamość,
Poland). Body mass (BM) and percent body fat $(\mathrm{BF} \%)$ were then measured with a four-electrode bioimpedance body analyzer (Tanita BC-418 MA, Tanita Corp., Tokyo, Japan). All anthropometric measurements were conducted by the same qualified researcher and in compliance with the standards recommended by the International Society for Advancement of Kinanthropometry (ISAK).

\section{Measures}

Sprint performance

A $30-\mathrm{m}$ sprint was performed on an indoor synthetic track from a stationary start position (the preferred foot forward). The test was preceded by a 10-min dynamic warm-up involving 5 min of sub-maximal multi-directional running (including several accelerations) and 5 min of light dynamic exercise targeting the main muscle groups of the lower extremities. Two trials were performed with $3 \mathrm{~min}$ of active recovery between trials. A photocell timing system (Witty System, Microgate Srl, Bolzano, Italy) was used to measure 5- and 30-m times ( $\mathrm{T} 5 \mathrm{~m}$ and $\mathrm{T} 30 \mathrm{~m}$, respectively) to the nearest $0.01 \mathrm{~s}$, with the best time registered for further analysis.

Lower extremity dynamic strength

The 1RM test for the half squat was performed in accordance with a standardized protocol (Baechle et al., 2008). The test was preceded by a warm-up involving three sets of half squats with progressive loads (40-70-85\% of predicted 1RM) with a decreasing number of repetitions (10-6-3). The half back squat was performed from an upright position with the barbell set on the shoulders and firmly grasped with both hands. The participant then squatted to $90^{\circ}$ of knee flexion and returned to the upright position with fully extended legs. After two successful repetitions with a load approximately $5 \%$ below the predicted 1RM, an additional load of $1 \mathrm{~kg}$ was added and the procedure was repeated. If the second repetition could not be completed the test was terminated and the corresponding load was accepted as the individual's 1RM. A rest interval of $3 \mathrm{~min}$ was provided between the attempts. On average, 1RM was achieved within 4-6 attempts. Absolute and relative values (normalized to $\mathrm{BM}$ ) were used for analysis.

Lower extremity isometric strength

$$
\text { A maximal voluntary isometric }
$$


contraction (MVIC) of the knee extensors was performed with the dominant leg (designated as the preferred kicking leg). The participant performed a 5-min warm-up on a cycle ergometer before being positioned in an isokinetic dynamometer using a standardized protocol (BIODEX-3 Pro, Biodex Medical Systems Inc., Shirley, NY, USA). Straps were used to secure the chest, hips, thigh, and distal femur. The lateral femoral epicondyle was aligned with the axis of rotation of the dynamometer and the knee was fixed at $90^{\circ}$ of flexion. The participant then performed a series of practice trials at $50 \%, 75 \%$, and $100 \%$ perceived maximal effort before performing three 3-s MVICs each separated by 30 $\mathrm{s}$ of rest. The examiner provided verbal motivation to ensure maximal effort. Peak kneeextensor torque $(\mathrm{Nm})$ in the three trials was recorded and both absolute and relative (normalized to BM) values were used for analysis.

Countermovement jump test

Countermovement jump (CMJ) testing was performed on a force plate (JBA-Z, Staniak, Warsaw, Poland). Testing was preceded by a 5min warm-up of 3-4 submaximal CMJs and dynamic exercise (no static stretching exercises were allowed). The participant was instructed to complete the CMJ with an arm swing by squatting down to approximately $90^{\circ}$ of knee flexion and jumping upwards as high as possible via a maximal concentric contraction. Participants performed two practice jumps followed by five maximal jumps separated by 6-s rest intervals. Vertical jump height (CMJheight) was determined as the change in height of the participant's center of mass from the moment of take-off to the maximum jump height (predicted error $=3.0 \%$ ). Maximal power output (CMJpower) was defined as the peak force generated during the upward movement. The highest values obtained in the five attempts were registered for further analysis. Wingate Anaerobic Test

Anaerobic power was determined by the 10-s version of the Wingate Anaerobic Test (WAnT) (Zając et al., 1999). The test was performed on a calibrated cycle ergometer (Monark Ergomedic 874-E, Monark Exercise AB, Vansbro, Sweden) adjusted to each participant (saddle height configured to allow 5-10 $0^{\circ}$ knee flexion). Testing was preceded by a standardized 5-min warm-up which consisted of cycling on the ergometer at a constant workload $(2.0 \mathrm{~W} / \mathrm{kg}$ of $\mathrm{BM})$ and cadence ( $\sim 60 \mathrm{rev}$ per min) that included three all-out $2-3 \mathrm{~s}$ sprints ( $\sim 90 \mathrm{rev}$ per $\mathrm{min}$ ) to elicit a heart rate between 150 and 160 beats per min. Participants then rested for $5 \mathrm{~min}$ before performing the WAnT. The test involved cycling as fast as possible for $10 \mathrm{~s}$ at a constant workload

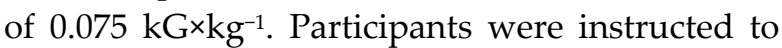
remain seated during the test and verbally encouraged by the examiner to provide maximal effort for the entire test duration. Real-time power output was recorded at a frequency of $1000 \mathrm{~Hz}$ and peak power was determined. Absolute maximal power output $\left(\mathrm{P}_{\max }\right)$ was then calculated, defined as the average value of power during the time at or above $97.7 \%$ of peak power, as well as $\mathrm{P}_{\max }$ normalized to $\mathrm{BM}\left(\mathrm{P}_{\max } / \mathrm{BM}\right)$.

Aerobic capacity test

On the second day of testing a field-based assessment of aerobic performance was administered via a $20-\mathrm{m}$ multi-stage shuttle run test (MST) in accordance with Léger and Lambert (1982). The MST was performed on a nonslip surface $(20 \times 40 \mathrm{~m})$ and involved running back and forth between two lines set $20 \mathrm{~m}$ apart at a pace dictated by an audio signal (timed "beep"). Each 20-m run completed before the sound of the "beep" was considered as the completion of one shuttle. Participants performed the test in small groups of no more than four athletes to promote rivalry and enhance motivation to run as fast as possible (verbal encouragement was also provided by the examiners). Participants first completed a standardized warm-up consisting of jogging for 3 min followed by multiple dynamic stretching exercises of the lower extremities. To complete the first stage of the shuttle, a running

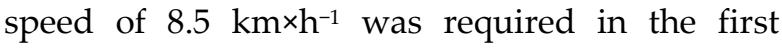
minute to complete the $20 \mathrm{~m}$ distance before the "beep". Running pace was progressively increased every $1 \mathrm{~min}$ by $0.5 \mathrm{~km} / \mathrm{h}$. The participant was cautioned if they did not cross the end line before the "beep" and the test was terminated for that individual if he failed to cross the line on two successive shuttles or until volitional exhaustion. The number of completed shuttles was calculated and the distance covered $(20 \mathrm{~m} \times$ number of completed shuttles) was treated as a measure of aerobic endurance performance. Heart rate (HR) was continuously recorded every $5 \mathrm{~s}$ during the test using short- 
range telemetry (Polar S 710, Polar Electro Oy, Helsinki, Finland) and HRmax was determined. $\mathrm{VO}_{2 \max }$ was predicted based on the equation: $Y=-$ $32.678+6.592$ MAS (Léger and Gadoury, 1989), where MAS is the maximal aerobic speed attained in the MST and $Y$ is the predicted $\mathrm{VO}_{2 \max }$ ( $\mathrm{mL} / \mathrm{kg} / \mathrm{min})$.

Blood lactate

Blood samples were drawn from the fingertips to $10-\mu \mathrm{L}$ heparinized capillary tubes to determine blood lactate concentration (BLa) $5 \mathrm{~min}$ before the MST (resting BLa) and within $3 \mathrm{~min}$ after terminating the MST (post-exercise BLa). BLa was immediately assayed by the amperometric enzymatic technique (LP-420, Dr Bruno Lange, $\mathrm{GmbH}$, Berlin, Germany). All blood samples were analyzed in duplicate and the mean of the two samples was used for statistical analysis.

\section{Statistical analysis}

All variables are presented as mean $\pm S D$. The Shapiro-Wilk test was used to confirm the normality of the data set. Pearson's productmoment correlation coefficients $(r)$ were calculated to determine the multiple bivariate correlations among the training-related values. Correlations were interpreted in accordance with the following scale of magnitude (Hopkins et al., 2009): $\leq 0.1$ (trivial); $>0.1-0.3$ (small); $>0.3-0.5$ (moderate); >0.5-0.7 (large); >0.7-0.9 (very large); and $>0.9-1.0$ (nearly perfect/perfect). Coefficients of determination $\left(r^{2}\right)$ were used to interpret the meaningfulness of the relationships. The level of significance was set at 0.05 in all procedures. The sample size was estimated a priori with standalone power analysis software $\left(G^{*}\right.$ Power software v. 3.1; University of Dusseldorf, Dusseldorf, Germany). A total of 19 subjects were required assuming an alpha level of 0.05 and statistical power (beta) of $\geq 0.90$ to detect a moderate $r^{2}$ value. All calculations were performed with a statistical software package (Statistica v. 10.1, StatSoft Inc., Tulsa, OK, USA) and spreadsheet software (Office Excel 2010, Microsoft Corporation, Redmond, WA, USA).

\section{Results}

Descriptive anthropometric and athletic performance variables are presented in Table 1, whereas performance and physiological variables determined in the MST are presented separately in Table 2. Test-retest intraclass correlation coefficients (ICCs) were calculated for performance variables and revealed high reliability: T5m (0.93), T30m (0.89), 1RM half back squat (0.94), knee-extensor MVIC (0.88), CMJheight (0.90), CMJ power (0.92), $P_{\max }(0.91)$, and distance covered in the MST (0.87).

An intercorrelation matrix is depicted in Table 3, where only multiple and at least moderate correlations found in the initial correlation analysis are presented. The strongest positive relationships were observed between post-exercise BLa and distance covered in the MST $\left(r=0.819, r^{2}=0.672, p<0.001\right)$ and $\mathrm{VO}_{2 \max }(r=$ $0.787, r^{2}=0.619, p<0.001$ ) (Figure 1). There was also a moderate correlation between post-exercise BLa and HRmax $\left(r=0.408, r^{2}=0.166, p<0.05\right)$ and a very high correlation between the distance covered in the MST and $\mathrm{VO}_{2 \max }\left(r=0.950, r^{2}=\right.$ $0.903, p<0.001$ ).

Further analysis revealed a moderate positive correlation between $\mathrm{T} 5 \mathrm{~m}$ and T30m $(r=$ $\left.0.497, r^{2}=0.247, p<0.05\right)$ and a large negative correlation between sprint times and 1RM half back squat/BM ( $r=-0.510$ to $-0.570, r^{2}=0.260$ 0.325 , both $p<0.01)$ (Figure 2) and $\mathrm{P}_{\max } / \mathrm{BM}(r=-$ $\left.0.501, r^{2}=0.251, p<0.01\right)$. T30m most strongly and negatively correlated with CMJheight $\left(r=-0.744, r^{2}=\right.$ $0.554, p<0.001)$, but also with absolute and relative 1RM half back squat $\left(r=-0.572\right.$ to $-0.570, r^{2}$ $=0.325-0.327$, both $p<0.01$ ). Additionally, this variable was similarly correlated with absolute and relative $P_{\max }\left(r=-0.448, r^{2}=0.201, p<0.05\right.$ and $r=-0.540, r^{2}=0.292, p<0.01$, respectively).

WAnT-determined $\mathrm{P}_{\max }$ showed a very high correlation between absolute $P_{\max }$ and kneeextensor MVIC ( $\left.r=0.827, r^{2}=0.684, p<0.001\right)$ and significant correlations between absolute $P_{\max }$ and 1 RM half squat $\left(r=0.674, r^{2}=0.454, p<0.001\right)$ and $\mathrm{CMJ}_{\text {power }}\left(r=0.579, r^{2}=0.335, p<0.01\right)$ (Figure 3). Regarding relative $\mathrm{P}_{\max } / \mathrm{BM}$, we found a very large correlation with CMJheight $\left(r=0.703, r^{2}=0.494, p<\right.$ $0.001)$ and large correlations with MVIC/BM $(r=$ $\left.0.658, r^{2}=0.433, p<0.001\right)$ and $\mathrm{CMJ}$ power $/ \mathrm{BM}(r=$ $\left.0.614, r^{2}=0.377, p<0.001\right)$. There were also large correlations between $\mathrm{P}_{\max } / \mathrm{BM}$, sprint times, and 1RM half back squat/BM $\left(r=-0.501-0.558, r^{2}=\right.$ $0.251-0.311$, all $p<0.01$ ). We also identified a large inverse relationship between $\mathrm{CM}$ height and $\mathrm{T} 30 \mathrm{~m}$ $\left(r=-0.744, r^{2}=0.554, p<0.001\right)$ and a large positive correlation between CMJheight and MVIC/BM ( $r=$ $0.702, p<0.001)$. A large positive relationship was 
also observed between $1 \mathrm{RM}$ half back squat/BM and $\mathrm{CMJheight}\left(r=0.603, r^{2}=0.366, p<0.01\right)$ as well as between $1 \mathrm{RM}$ half back squat/BM and
MVIC/BM $\left(r=0.633, r^{2}=0.402, p<0.001\right)$ (Figure 4).

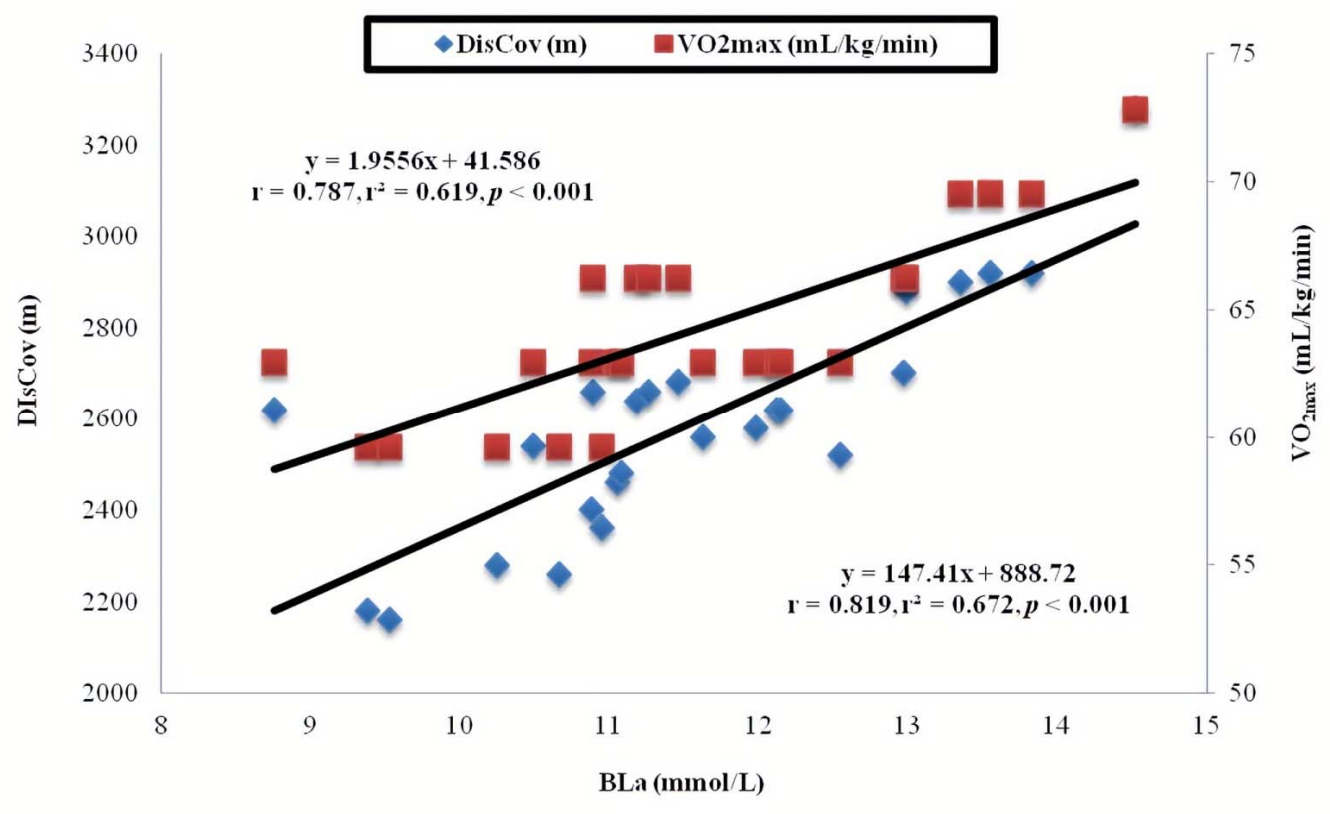

Figure 1.

Relationships of post-exercise blood lactate concentration ( $B L a$ ) to distance covered in the MST (DisCov) and predicted maximal oxygen uptake $\left(V_{2} \mathrm{z}_{\max }\right)$

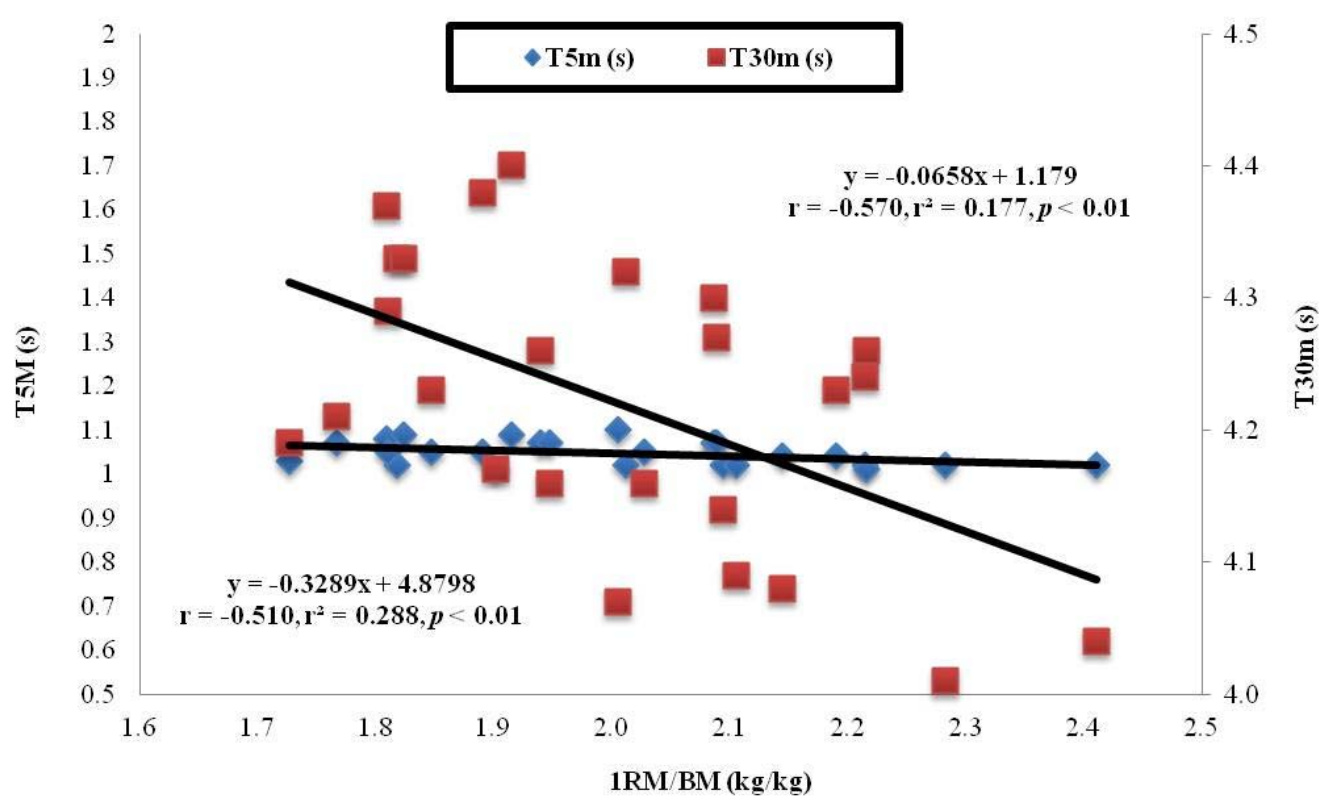

Figure 2.

Relationships of relative one repetition maximum half squat (1RM/BM) with 5-m (T5m) and $30-m$ sprint time (T30m) 


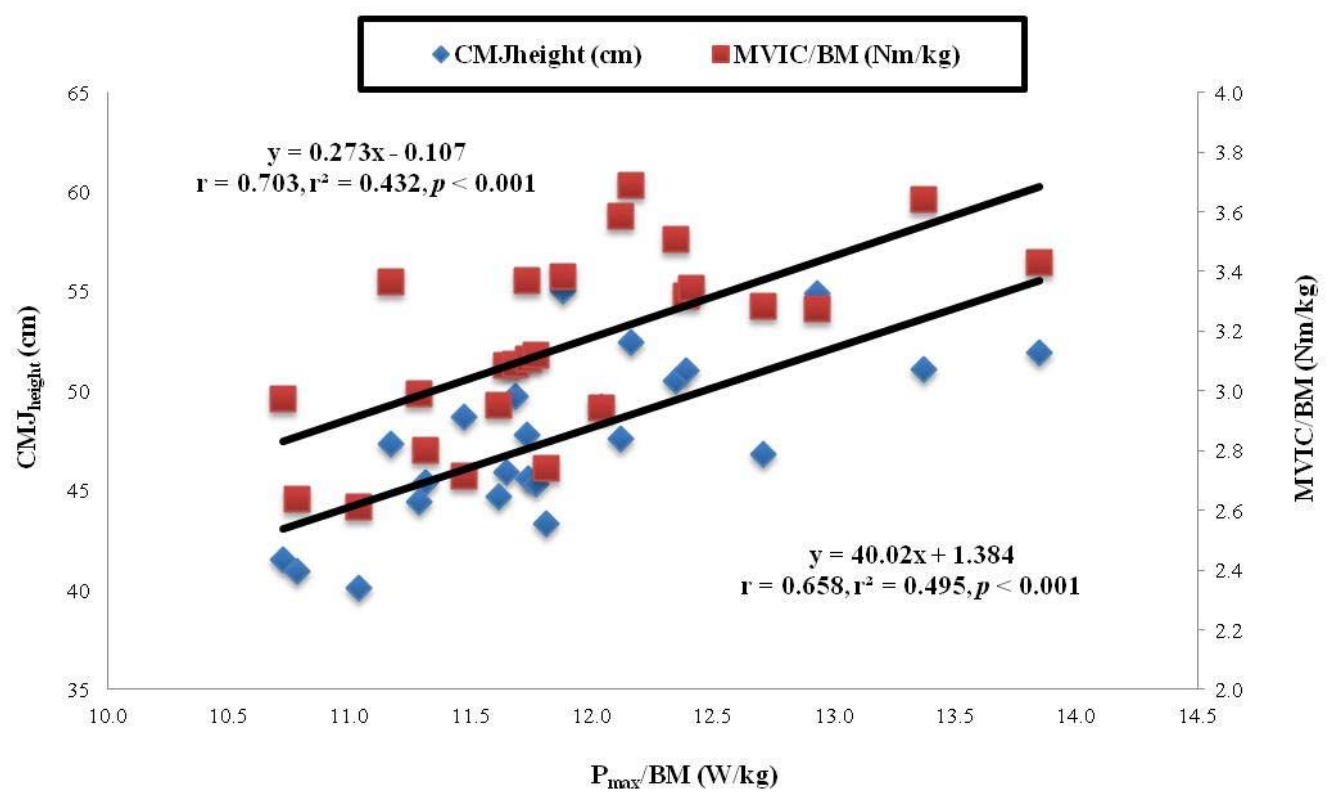

Figure 3.

Relationships of relative maximal power output ( $\left.P_{\max } / B M\right)$ to countermovement jump height (CM $J_{\text {height }}$ ) and relative maximal voluntary isometric contraction (MVIC/BM)

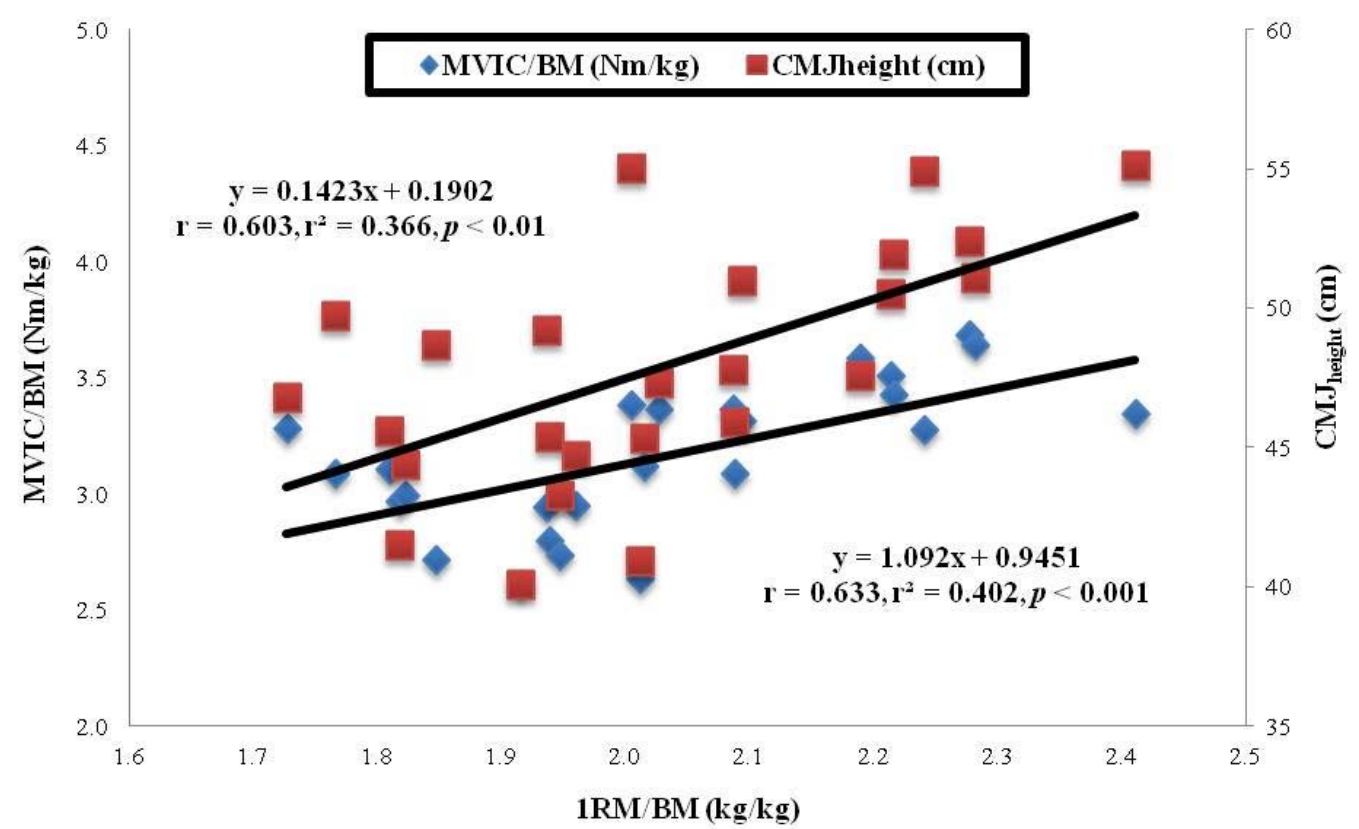

Figure 4.

Relationships of relative one repetition maximum half squat (1RM/BM) to relative maximal voluntary isometric contraction (MVIC/BM) and countermovement jump height (CMJheight) 
Table 1

Anthropometric and fitness variables

\begin{tabular}{lcc}
\hline Variables & Mean $\pm S D$ & Range (Min - Max) \\
\hline Body height $(\mathrm{cm})$ & $180.6 \pm 5.45$ & $172.4-190.2$ \\
Body mass $(\mathrm{kg})$ & $75.2 \pm 5.92$ & $65.1-85.7$ \\
Body fat $(\%)$ & $14.0 \pm 3.47$ & $4.6-19.3$ \\
T5m (s) & $1.04 \pm 0.03$ & $1.01-1.09$ \\
T30m (s) & $4.22 \pm 0.11$ & $4.01-4.42$ \\
CMJheight $(\mathrm{cm})$ & $46.0 \pm 4.6$ & $39.0-5.73$ \\
CMJpower $(\mathrm{W})$ & $2914.9 \pm 303.75$ & $2156-3532$ \\
CMJpower $/ \mathrm{BM}(\mathrm{W} / \mathrm{kg})$ & $38.7 \pm 4.38$ & $29.7-47.2$ \\
$1 \mathrm{RM}(\mathrm{kg})$ & $150.1 \pm 15.12$ & $130-185$ \\
$1 \mathrm{RM} / \mathrm{BM}(\mathrm{kg} / \mathrm{kg})$ & $2.0 \pm 0.18$ & $1.7-2.4$ \\
MVIC $(\mathrm{Nm})$ & $237.6 \pm 31.47$ & $178.2-302.8$ \\
MVIC/BM $(\mathrm{Nm} / \mathrm{kg})$ & $3.16 \pm 0.31$ & $2.6-3.7$ \\
$P_{\max }(\mathrm{W})$ & $896.8 \pm 84.63$ & $704-1052$ \\
$P_{\max } / \mathrm{BM}(\mathrm{W} / \mathrm{kg})$ & $11.92 \pm 0.75$ & $10.73-13.85$ \\
\hline
\end{tabular}

Abbreviations: T5m-5-m Sprint Time; T30m-30-m sprint time; CMJheight - Countermovement Jump Height;

CMJpower - Countermovement Jump Power; 1RM - One Repetition Maximum of the Half Squat; MVIC - Maximal Voluntary Isometric Contraction of the Knee Extensor; $P_{\max }-$ Maximal Power Output determined in the Wingate Anaerobic Test

\section{Table 2}

Athletic performance and physiological variables determined in the 20-m multi-stage shuttle run (MST)

\begin{tabular}{lcc}
\hline Variables & Mean $\pm S D$ & Range (Min - Max) \\
\hline Distance covered $(\mathrm{m})$ & $2595.2 \pm 257.64$ & $2160-3280$ \\
Maximal aerobic speed $(\mathrm{km} / \mathrm{h})$ & $14.7 \pm 0.54$ & $14.0-16.0$ \\
HRmax $(\mathrm{b} /$ minute) & $195.4 \pm 5.35$ & $185-203$ \\
Resting BLa (mmol/L) & $1.54 \pm 0.47$ & $0.72-2.14$ \\
Post-exercise BLa (mmol/L) & $11.58 \pm 1.43$ & $8.76-13.82$ \\
Time to exhaustion (min) & $14.0 \pm 1.01$ & $12.0-16.5$ \\
VO2max $(\mathrm{mL} / \mathrm{kg} / \mathrm{min})$ & $64.2 \pm 3.56$ & $59.6-72.8$ \\
\hline
\end{tabular}

Abbreviations: HRmax - Maximal Heart Rate; BLa - Blood Lactate Concentration;

$\mathrm{VO}_{2 \max }$ - Maximal Oxygen Uptake 
Table 3

Intercorrelation matrix ( $r$-values) for selected physiological and fitness variables

\begin{tabular}{|c|c|c|c|c|c|c|c|c|c|c|c|c|c|c|}
\hline Variables & T30m & $1 \mathrm{RM}$ & $\begin{array}{c}\text { 1RM/ } \\
\text { BM }\end{array}$ & MVIC & $\begin{array}{c}\text { MVIC/ } \\
\text { BM }\end{array}$ & CMJheight & $\mathrm{CMJ}_{\text {power }}$ & $\begin{array}{c}\mathrm{CMJ}_{\text {power }} / \\
\mathbf{B M}\end{array}$ & $\mathbf{P}_{\max }$ & $\mathbf{P}_{\max } / \mathbf{B M}$ & $\begin{array}{l}\text { Dis } \\
\text { Cov }\end{array}$ & $\mathrm{VO}_{2 \max }$ & $\mathbf{H R}_{\max }$ & BLa \\
\hline T5m & $0.497^{*}$ & -0.281 & $\begin{array}{c}-0.510 \\
+\end{array}$ & -0.229 & $-447^{*}$ & $-426^{*}$ & -0.221 & -0.340 & -0.169 & $-0.501+$ & -0.022 & -0.103 & -0.172 & -0.192 \\
\hline T30m & 1.000 & $\begin{array}{c}-0.572 \\
+\end{array}$ & $\begin{array}{c}-0.570 \\
+\end{array}$ & $\begin{array}{c}-0.486 \\
*\end{array}$ & $-0.596 \dagger$ & $-744 \ddagger$ & $-0.612 \ddagger$ & $-0.499^{*}$ & $-448^{*}$ & $-0.540+$ & 0.166 & 0.198 & -0.032 & -0.116 \\
\hline 1RM & & 1.000 & $0.684 \ddagger$ & $0.780 \ddagger$ & $0.629 \ddagger$ & $0.394^{*}$ & $0.408^{*}$ & 0.029 & $0.674 \ddagger$ & 0.371 & -0.070 & -0.073 & -0.219 & -0.002 \\
\hline 1RM/BM & & & 1.000 & 0.308 & $0.633 \ddagger$ & $0.603 \ddagger$ & 0.233 & $0.411^{*}$ & 0.121 & $0.558+$ & 0.049 & 0.049 & 0.077 & 0.192 \\
\hline MVIC & & & & 1.000 & $0.796 \ddagger$ & $0.398^{*}$ & $0.521 \dagger$ & 0.032 & $0.827 \ddagger$ & 0.379 & -0.136 & -0.140 & -0.150 & -0.031 \\
\hline $\begin{array}{l}\text { MVIC/ } \\
\text { BM }\end{array}$ & & & & & 1.000 & $0.702 \ddagger$ & $0.513 t$ & $0.433^{*}$ & $0.512 \dagger$ & $0.658 \ddagger$ & -0.063 & -0.069 & 0.102 & 0.143 \\
\hline CMJ Jeight & & & & & & 1.000 & $0.690 \ddagger$ & $0.778 \ddagger$ & 0.308 & $0.703 \ddagger$ & -0.341 & -0.316 & 0.372 & 0.002 \\
\hline $\mathrm{CMJ}_{\text {power }}$ & & & & & & & 1.000 & $0.741 \ddagger$ & $0.579+$ & $0.530+$ & $-0.414^{*}$ & -0.387 & -0.116 & -0.154 \\
\hline $\begin{array}{l}\mathrm{CMJ}_{\text {power }} / \\
\text { BM }\end{array}$ & & & & & & & & 1.000 & 0.025 & $0.614 \ddagger$ & -0.260 & -0.232 & 0.386 & 0.034 \\
\hline $\mathbf{P}_{\max }$ & & & & & & & & & 1.000 & $0.522+$ & -0.239 & -0.262 & -0.197 & -0.165 \\
\hline $\mathbf{P}_{\max } / \mathbf{B M}$ & & & & & & & & & & 1.000 & -0.172 & -0.203 & 0.207 & 0.043 \\
\hline DisCov & & & & & & & & & & & 1.000 & $0.950 \ddagger$ & 0.078 & $0.819 \ddagger$ \\
\hline $\mathbf{V O}_{2 \max }$ & & & & & & & & & & & & 1.000 & 0.126 & $0.787 \ddagger$ \\
\hline $\mathbf{H R}_{\max }$ & & & & & & & & & & & & & 1.000 & $0.408^{*}$ \\
\hline BLa & & & & & & & & & & & & & & 1.000 \\
\hline
\end{tabular}

${ }^{*} p \leq 0.05,+p \leq 0.01, \neq p \leq 0.001 ; B M-$ Body Mass; T5m-5 meter sprint time; T30m-30 meter sprint time;

1RM - One Repetition Maximum; 1RM/BM - Relative One Repetition Maximum;

MVIC - Maximal Voluntary Isometric Contraction; CMJheight - Countermovement Jump Height;

CMJpover - Countermovement Jump Power; CMJpower/BM - Relative Countermovement Jump Power;

$P_{\max }$ - Maximal Power Output; $P_{\max } / B M$ - Relative Maximal Power Output;

DisCov - Distance Covered during the MST; VOrmax - Maximal Oxygen Uptake;

HRmax - Maximal Heart Rate; BLa - Post-Exercise Blood Lactate Concentration 


\section{Discussion}

The aim of the present investigation was to re-examine previously reported relationships between multiple physiological and athletic performance variables in professional soccer players. Although correlational studies can only provide insight into the associations between variables and not the cause-effect relationship, the results of this study support previously reported strong relationships between maximal isometric or functional strength, sprinting ability, WAnTdetermined maximal power output, and jumping performance in highly-trained soccer players (Chelly et al., 2010; Little and Williams, 2005; McBride et al., 2009; Requena et al., 2009; Wisloff et al., 2004).

An increase in 1RM is usually associated with improved power output and performance, with several studies involving elite soccer players finding that resistance training-induced beneficial neural adaptations enhanced not only strength (quantified by 1RM), but also sprinting speed and jumping height without any change in BM (Hoff, 2005) or other studies in which athletes with greater maximum strength showed higher jump heights (Gourgoulis et al., 2003). Our observations confirm this maximal strength/power relationship although we expected a stronger association between the 1RM half squat and $\mathrm{CMJ}_{\text {power. }}$ We instead observed a moderate correlation between these two variables $(r=0.408, p<0.05)$, a weak although significant correlation between 1RM and CMJheight $(\mathrm{r}=0.394, \mathrm{p}<0.05)$, and no correlation

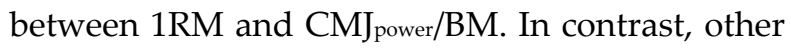
studies have found this relationship to be unclear, such as Requena et al. (2009) who found no significant correlation between the traditional squat $1 \mathrm{RM}$ and CMJ performance.

It was suggested by Bret et al. (2002) that CMJ testing represented a suitable measure of predicting performance in sprints over $30 \mathrm{~m}$. In agreement, Nesser et al. (1996) showed CMJ performance to have a significant negative relationship with $40 \mathrm{~m}$ sprint time $(\mathrm{r}=-0.464, \mathrm{p}<$ 0.05). However, Chelly et al. (2010) reported that the squat jump (SJ) was a stronger predictor of acceleration and sprint performance at shorter distance sprints (e.g. $5 \mathrm{~m}$ ). Cronin et al. (2005) found moderate to large correlations between SJ performance (height and relative power output) and $\mathrm{CMJ}_{\text {height }}$ and three sprint measures $(r=-0.43$ to $-0.66, p<0.05)$ and found that SJ performance and $\mathrm{CMJ}_{\text {height }}$ were significantly greater in athletes with faster sprint times. While the $\mathrm{CMJ}_{\text {power }}$ relationship with running velocity has been observed over longer sprint distances (Chelly et al., 2010), we identified a large inverse relationship between $\mathrm{CMJ}_{\text {power }}$ and $\mathrm{T} 30 \mathrm{~m}$ ( $\mathrm{r}=$ $-0.612, p<0.001)$ and an even stronger correlation between $\mathrm{CMJ}$ height and T30m ( $\mathrm{r}=-0.744, \mathrm{p}<0.001)$. On the other hand, a number of factors such as lower reliability of testing at very short distances and the static start position in the sprint test could explain the lack of the relationship between $\mathrm{CMJ}_{\text {power }}$ and $\mathrm{T} 5 \mathrm{~m}$. Additionally, only a moderate relationship observed between $\mathrm{CMJ}$ height and $\mathrm{T} 5 \mathrm{~m}$ $(\mathrm{r}=-0.426, \mathrm{p}<0.05)$ confirms this assumption. With respect to sprint performance, although we observed a moderate correlation between $\mathrm{T} 5 \mathrm{~m}$ and T30m $(\mathrm{r}=0.497, \mathrm{p}<0.05)$, other studies found very strong correlations between sprint times over different distances ( $\mathrm{r}=0.62-0.87)$ (Cronin et al., 2005; Harris et al., 2008; Little and Williams, 2005; Nesser et al., 1996). For example, Little and Williams (2005) showed a strong correlation between $10-\mathrm{m}$ sprint time and flying 20-m sprint time $\left(\mathrm{r}=0.623, \mathrm{r}^{2}=0.388, \mathrm{p}<0.001\right)$. Moreover, Nesser et al. (1996) used stepwise regression analysis to identify $10-\mathrm{m}$ sprint time as a predictor of $40-\mathrm{m}$ sprint performance $\left(\mathrm{r}=0.855, \mathrm{r}^{2}=0.731, \mathrm{p}\right.$ $<0.001)$. These clear and strong relationships are logical as a faster start and acceleration at the beginning of a sprint would translate to faster 30$\mathrm{m}$ and $40-\mathrm{m}$ sprint times. While sprint performance over various distances ( $<10 \mathrm{~m}$ vs. $>30$ $\mathrm{m})$ is considered by sports exercise specialists to require separate and specific strength qualities and therefore training methods (Delecluse et al., 1995; Young et al., 2001), it has been established that one of the main predictors of this quality is the ability to generate large ground reaction forces via lower-body muscle action by the quadriceps and hamstrings (Hunter et al., 2005; McBride et al., 2009). While some studies presented very low correlations between maximal strength and sprint times (Harris et al., 2008; Kukolj et al., 1999), the majority of investigations have reported strong relationships between these measures (Bissas and Havenetidis, 2008; Chelly et al., 2010; Comfort et al., 2014; Cronin et al., 2005; Dowson et al., 1998; McBride et al., 2009; Wisloff et al., 2004). Among these studies, Wisloff et al. (2004) found that 
elite soccer players with high $\mathrm{VO}_{2 \max }$ showed strong correlations between the 1RM half squat and $10-\mathrm{m}$ sprint $(\mathrm{r}=0.94, \mathrm{p}<0.001), 30-\mathrm{m}$ sprint $(\mathrm{r}$ $=0.71, \mathrm{p}<0.01)$, and $10-\mathrm{m}$ shuttle sprint performance $(\mathrm{r}=0.68, \mathrm{p}<0.05)$. The unusually high correlation between 1RM half back squat and 10-m sprint time may be attributed to the specific design of the study and incorporation of a highly standardized training protocol.

Analysis of the correlations between strength and sprint performance measures revealed that relative strength was more strongly associated with short sprint performance. While the correlation between $\mathrm{T} 5 \mathrm{~m}$ and the absolute 1RM half squat was low and non-significant $(r=-$ $0.281, p>0.05)$, a large negative relationship was found between T5m and the 1RM half squat/BM ( $\mathrm{r}$ $=-0.510, p<0.01)$. It is worth noting that multiple linear regression analyses showed that $\mathrm{T} 5 \mathrm{~m}$ and T30m accounted for only $29 \%$ and $18 \%$ of the total variance in 1RM half squat/BM. Nevertheless, the stronger and negative correlation between sprint performance and strength relative to BM confirms previous findings by Wong et al. (2009) who reported that $\mathrm{BM}$ was negatively correlated with $30-\mathrm{m}$ sprint time $(\mathrm{r}=-0.54, \mathrm{p}<0.001)$. On the contrary, Malina et al. (2004) showed that BM was the most significant predictor of $30-\mathrm{m}$ sprint performance in young soccer players, whereas Harris et al. (2008) showed that BM was moderately correlated with $10-\mathrm{m}$ sprint time $(\mathrm{r}=$ $0.40, \mathrm{p}<0.01)$ and largely correlated with $30-$ and $40-\mathrm{m}$ sprint time $(\mathrm{r}=0.63, \mathrm{p}<0.01)$. However, the aforementioned authors also reported a nonsignificant correlation between the 1RM machine squat and 10-m and 40-m sprint times $(r=-0.200$ and $r=-0.140$, respectively). In contrast, McBride et al. (2009) obtained significant correlations between 1RM squat/BM and 40-yard sprint $(r=-$ $0.605, \mathrm{p}=0.010)$ and 10-yard sprint times $(\mathrm{r}=-$ $0.544, p=0.024)$. In a more recent study, absolute $1 \mathrm{RM}$ back squat strength showed the strongest correlations with 5-m sprint time $(\mathrm{r}=-0.596, \mathrm{p}<$ 0.001 ), whereas relative $1 R M$ back squat strength was more strongly correlated with $20-\mathrm{m}$ sprint time $(\mathrm{r}=-0.672, \mathrm{p}<0.001)$ (Comfort et al., 2014). In another study (Requena et al., 2011), negative correlations were found between relative squat strength and sprint times across a range of distances $(r=-0.49$ to $-0.59, \mathrm{p}<0.05)$, yet there were no significant relationships with absolute strength. Therefore, the results may suggest that relative strength produced either with half squat exercise or the vertical jump is an important variable to explain long sprint performance in soccer players.

When considering the MVIC, it is important to note that the half squat is a multijoint movement that reflects functional strength whereas a MVIC is an isolated single-joint isometric strength measure which shows a weaker association with total-body dynamic movements such as sprinting. In this regard, we observed a significant negative correlation between $\mathrm{T} 30 \mathrm{~m}$ and both absolute and relative MVIC ( $\mathrm{r}=-0.486, \mathrm{p}$ $<0.05$ and $\mathrm{r}=-0.596, \mathrm{p}<0.01$, respectively). This inverse association was also observed between T5m and MVIC/BM ( $\mathrm{r}=-0.447, \mathrm{p}<0.05)$. Considering the relative 1RM half back squat and MVIC, the correlations were very similar and the coefficients of determination accounted for 20$26 \%$ of variance in $\mathrm{T} 5 \mathrm{~m}$ and $32-36 \%$ of variance in T30m. Previous research by Dowson et al. (1998) reported that concentric knee extension torque at $4.19 \mathrm{rad} / \mathrm{s}$ and the time to reach $15 \mathrm{~m}$ and $35 \mathrm{~m}$ were significantly correlated $(r=-0.518$ and $r=-$ 0.688 , respectively). In a similar study by Bissas and Havenetidis (2008), isometric leg extensions were performed by trained males and the time to reach $60 \%$ of maximum force was significantly correlated with maximum running velocity $(r=$ 0.730), with the coefficient of determination accounting for $53 \%$ of the variance in the data. Kin-Isler et al. (2008) showed a weak albeit significant correlation between $240 /{ }^{\circ} \mathrm{s}^{-1}$ knee flexion strength and the percentage of performance decrement from 10-20 m sprint $(\mathrm{r}=$ $0.381, \mathrm{p}<0.05)$, although the authors showed no significant relationships between knee extension and flexion strength evaluated at 60, 150 and $240 /{ }^{\circ} s$ and single-sprint and repeated-sprint ability.

The WAnT has been increasingly employed to evaluate anaerobic fitness of soccer players and is considered a valid predictor of short-distance sprint performance (Magal et al., 2009; Meckel et al., 2009). In agreement with the correlations we observed between $\mathrm{P}_{\max } / \mathrm{BM}$ and $\mathrm{T} 5 \mathrm{~m}(\mathrm{r}=-0.501, \mathrm{p}<0.01)$ and between both $\mathrm{P}_{\max }$ and $\mathrm{P}_{\max } / \mathrm{BM}$ with T30m $(\mathrm{r}=-0.448, \mathrm{p}<0.05$ and $\mathrm{r}$ $=-0.540, \mathrm{p}<0.01$, respectively), Meckel et al. 
(2009) showed that WAnT-determined mean $P_{\max }$ was significantly correlated with the fastest sprint and the total sprint time of a $6 \times 40 \mathrm{~m}$ protocol $(\mathrm{r}=$ -0.42 and -0.45 , respectively) and with the total sprint time of a $12 \times 20 \mathrm{~m}$ protocol $(\mathrm{r}=-0.47)$. In turn, Aziz and Chuan (2004) reported high correlations between the fastest sprint time in a RSA test and WAnT-determined $P_{\max }$ and $\mathrm{P}_{\max } / \mathrm{BM}$ $(\mathrm{r}=-0.053$ and $\mathrm{r}=-0.63$, both $\mathrm{p}<0.01$, respectively). Apart from the $P_{\max }$ and sprinting correlations that were identified, we also found a large positive relationship between $\mathrm{P}_{\max } / \mathrm{BM}$ and all three CMJ indices $(r=0.530-0.703)$, with the very large correlation for $P_{\max } / \mathrm{BM}$ and $\mathrm{CM} \mathrm{Jheight}_{\mathrm{r}} \mathrm{r}$ $=0.703, \mathrm{p}<0.001)$. However, given the large positive relationship between $\mathrm{CMJ}_{\text {power }}$ and WAnT-determined $\mathrm{P}_{\max }(\mathrm{r}=0.579, \mathrm{p}<0.01)$, it may be more beneficial for coaches to omit WAnT testing as it is not a soccer-specific task and focus on jumping and running assessment when working with elite soccer players.

Both predicted $\mathrm{VO}_{2 \max }$ and $\mathrm{HR}_{\max }$ measured in the MST presented no significant relationships with any of the strength, sprint, anaerobic, or jumping performance variables. However, there is some evidence showing significant albeit low to moderate correlations between aerobic fitness $\left(\mathrm{VO}_{2 \max }, \mathrm{MAS}\right.$, distance covered) and RSA in professional soccer players (Aziz et al., 2000; Bishop and Edge, 2006). In a study involving elite soccer players, da Silva et al. (2010) reported that the RSA is more strongly correlated with the onset of blood lactate accumulation (OBLA) and $\mathrm{VO}_{2 \max }$ than other physiological and aerobic indices, although they found that the strongest predictor of the RSA was anaerobic power (quantified as the fastest individual sprint time). In the study of Aziz et al. (2000), no significant correlations were established between the fastest $40-\mathrm{m}$ sprint time in the RSA test $\left(8 \times 40 \mathrm{~m}\right.$ sprints) and $\mathrm{VO}_{2 \max }(\mathrm{r}=-0.080, \mathrm{p}>$ 0.05 ) although a moderate correlation was found between total sprint time in the RSA and $\mathrm{VO}_{2 \max }(\mathrm{r}$ $=-0.323, p<0.05)$. The only significant correlation regarding predicted $\mathrm{VO}_{2 \max }$ was with the distance covered in the MST. As $\mathrm{VO}_{2 \max }$ was predicted by incorporating maximal running speed achieved during the MST, which reflects the distance covered, this was expected (Léger and Lambert, 1982; Ramsbottom et al., 1988). Previous studies have validated the MST as a predictor of $\mathrm{VO}_{2 \max }$ with a moderate correlation demonstrated between measured $\mathrm{VO}_{2 \max }$ (in $\mathrm{L} / \mathrm{min}$ and $\mathrm{mL} / \mathrm{kg} / \mathrm{min})$ and MST performance $(\mathrm{r}=$ 0.43 and $0.54, \mathrm{p}<0.05$, respectively) (Aziz et al., 2007). The high correlation of post-exercise BLa and distance covered in the MST $(\mathrm{r}=0.819, \mathrm{p}<$ 0.001 ) found in our study might indicate the predominance of anaerobic glycolysis in the test and not aerobic metabolism. Probably players that covered a greater distance performed at higher intensity and not necessarily had higher aerobic capacity. One must note that the use of BLa to provide a modulation to the intensity of exercise suggests that it is reflective of the overall physiological stress imposed on the athlete at a given exercise intensity. Alternatively, as BLa is the net result of lactate production in the muscle, its release from the muscle and uptake by other tissues, a change in BLa may reflect any of those factors independently and not necessarily the overall physiological stress of exercising at that intensity.

\section{Limitations of the study}

This study has some limitations. Due to limited access to professional soccer players, the present study is hampered by small sample size and thus needs to be confirmed in a larger sample, although many of the associations were quite robust. Furthermore, in statistical correlation analyses strong relationships are typically obtained with a sample of heterogeneous subjects. However, our subjects possessed homogenous characteristics (similar age, physical features, training experience and training regimen). Moreover, subjects were selected from a single squad. The conducted correlation analyses also do not take into account biochemical variables (except for blood lactate concentration) involved in a given relationship. Therefore, extra caution is clearly warranted when interpreting the present study's findings until confirmatory studies with more subjects and more sophisticated testing methods are obtained.

\section{Conclusions}

The current study demonstrates that elite soccer players with greater lower body strength (quantified by the MVIC of the knee extensor and the 1RM half squat) present better sprint (5-m and 30-m times) and CMJ performance. The higher correlation coefficients found between T30m and the physiological and athletic measures suggest 
the greater application of this sprint distance than $\mathrm{T} 5 \mathrm{~m}$ when assessing sprint performance. The strong relationships we observed between lower body strength and both sprint times suggest the incorporation of soccer-specific resistance training to develop lower body musculature and therefore maximize sprint performance. Progress in this realm can be monitored by free-weight half squat exercise.

Our results also support the use of relative measures (normalized to body mass) when comparing strength variables with sprint performance, CMJ indices or anaerobic power. Furthermore, considering the correlations we observed between maximal power output obtained in the WAnT and maximal power output in the CMJ test, it is suggested that coaches administer tests that assess jumping and linear sprint performance rather than the cyclingspecific WAnT. The lack of correlations between MST predicted $\mathrm{VO}_{2 \max }, \mathrm{HR}_{\max }$, and post-exercise BLa and the strength, anaerobic or jumping performance variables emphasizes the applicability of this test to quantify aerobic capacity, but not strength-speed abilities or anaerobic power.

\section{Acknowledgements}

We would like to thank for the patience and cooperation of the athletes and coaches during the execution of this study and the Department of Health Sciences of Olsztyn University for organizational support.

\section{References}

Aguiar M, Abrantes C, Maçãs V, Leite N, Sampaio J, Ibáñez S. Effects of intermittent or continuous training on speed, jump and repeated-sprint ability in semi-professional soccer players. TOSSJ, 2008; 1: 15-19

Aziz AR, Chia M, Teh KC. The relationship between maximal oxygen uptake and repeated sprint performance indices in field hockey and soccer players. J Sport Med Phys Fit, 2000; 40(3): 195

Aziz AR, Chuan TEH. Correlation between Tests of Running Repeated Sprint Ability and Anaerobic Capacity by Wingate Cycling in Multi-Sprint Sports Athletes. Int J Appl Sports Sci, 2004; 16(1): 14-22

Baechle TR, Earle RW, Wathen D. Essentials of Strength Training and Conditioning. National Strength and Conditioning Association (3rd ed.). T.R. Baechle and R.W. Earle, eds. Champaign, IL: Human Kinetics, 381-412; 2008

Bangsbo J, Mohr M, Krustrup P. Physical and metabolic demands of training and match-play in the elite football player. J Sports Sci, 2006; 24(07): 665-674

Bartlett R, Button C, Robins M, Dutt-Mazumder A, Kennedy G. Analysing team coordination patterns from player movement trajectories in soccer: methodological considerations. Int J Perf Anal Spor, 2012; 12(2): 398-424

Bishop D, Edge J. Determinants of repeated-sprint ability in females matched for single-sprint performance. Eur J Appl Physiol, 2006; 97(4): 373-379

Bissas AI, Havenetidis K. The use of various strength-power tests as predictors of sprint running performance. J Sports Med Phys Fit, 2008; 48: 49-54

Bret C, Rahmani A, Dufour AB, Messonnier L, Lacour JR. Leg strength and stiffness as ability factors in 100 m sprint running. J Sports Med Phys Fit, 2002; 42: 274-281

Castagna C, Abt G, D'Ottavio S. The relationship between selected blood lactate thresholds and match performance in elite soccer referees. J Strength Cond Res, 2002; 16(4): 623-627

Chelly MS, Fathloun M, Cherif N, Amar MB, Tabka Z, Van Praagh E. Effects of a back squat training program on leg power, jump, and sprint performances in junior soccer players. J Strength Cond Res, 2009; 23(8): 2241-2249

Comfort P, Stewart A, Bloom L, Clarkson B. Relationships between strength, sprint, and jump performance in well-trained youth soccer players. J Strength Cond Res, 2014; 28(1): 173-177.

Cronin JB, Hansen KT. Strength and power predictors of sports speed. J Strength Cond Res, 2005; 19: 349-357

da Silva JF, Guglielmo LG, Bishop D. Relationship between different measures of aerobic fitness and 
repeated-sprint ability in elite soccer players. J Strength Cond Res, 2010; 24(8): 2115-2121

Delecluse C, Van Coppenolle H, Willems E, Van Leemputte M, Diels R, Goris, M. Influence of high resistance and high-velocity training on sprint performance. Med Sci Sports Exerc, 1995; 27: 1203-1209

Dowson MN, Nevill ME, Lakomy HK, Nevill AM, Hazeldine, RJ. Modelling the relationship between isokinetic muscle strength and sprint running performance. J Sport Sci, 1998; 16: 257-265

Dupont G, Akakpo K, Berthoin, S. The effect of in-season, high-intensity interval training in soccer players. J Strength Cond Res, 2004; 18(3): 584-589

Glaister M. Multiple sprint work: physiological responses, mechanisms of fatigue and the influence of aerobic fitness. Sports Med, 2005; 35: 757-777

Gourgoulis V, Aggeloussis N, Kasimatis P, Mavromatis G, Garas A. Effect of submaximal half squats warmup program on vertical jump ability. J Strength Cond Res, 2003; 17: 342-344

Harris NK, Cronin JB, Hopkins WG, Hansen KT. Relationship between sprint times and the strength/power outputs of a machine squat jump. J Strength Cond Res, 2008; 22: 691-698

Hoff J, Helgerud J. Endurance and strength training for soccer players: Physiological considerations. Sports Med, 2004; 34: 165-180

Hoff J. Training and testing physical capacities for elite soccer players. J Sports Sci, 2005; 23(6): 573-582

Hopkins W, Marshall S, Batterham A, Hanin J. Progressive statistics for studies in sports medicine and exercise science. Med Sci Sports Exerc, 2009; 41(1): 3

Hunter JP, Marshall RN, McNair PJ. Relationships between ground reaction force impulse and kinematics of sprint-running acceleration. J Appl Biomech, 2005; 21: 31-43

Kin-Isler A, Ariburun B, Ozkan A, Aytar A, Tandogan R. The relationship between anaerobic performance, muscle strength and sprint ability in American football players. Isokinet Exerc Sci, 2008; 16(2): 87-92

Krustrup P, Mohr M, Amstrup T, Rysgaard T, Johansen J, Steensberg A, Pedersen P, Bangsbo J. The Yo-Yo Intermittent Recovery Test: Physiological response, reliability and validity. Med Sci Sport Exer, 2003; 35: 697-705

Kukolj M, Ropret R, Ugarkovic D, Jaric S. Anthropometric, strength, and power predictors of sprinting performance. J Sports Med Phys Fit, 1999; 39: 120-122

Léger LA, Lambert J. A maximal multistage 20m shuttle run test to predict $\mathrm{VO}_{2 \max }$. Eur J Appl Physiol, 1982; 49: $1-5$

Léger L, Gadoury C. Validity of the $20 \mathrm{~m}$ shuttle run test with 1 min stages to predict VO2max in adults. J Canad Sci Sport, 1989; 14(1): 21-26

Little T, Williams AG. Specificity of acceleration, maximum speed, and agility in professional soccer players. J Strength Cond Res, 2005; 19(1): 76-78

Little T, Williams AG. Measures of exercise intensity during soccer training drills with professional soccer players. J Strength Cond Res, 2007; 21(2): 367-371

López-Segovia M, Marques M, Van den Tillaar R, González-Badillo J. Relationships between vertical jump and full squat power outputs with sprint times in U21 soccer players. J Hum Kinet, 2011; 30: 135-144

Magal M, Smith RT, Dyer JJ, Hoffman JR. Seasonal variation in physical performance-related variables in male NCAA division III soccer players. J Strength Cond Res, 2009; 23(9): 2555-2559

Malina RM, Eisenmann JC, Cumming SP, Ribeiro B, Aroso J. Maturity-associated variation in the growth and functional capacities of youth football (soccer) players 13-15 years. Eur J Appl Physiol, 2004; 91: $555-562$

McBride JM, Blow D, Kirby TJ, Haines TL, Dayne AM, Triplett NT. Relationship between maximal squat strength and five, ten, and forty yard sprint times. J Strength Cond Res, 2009; 23(6): 1633-1636

Meckel Y, Machnai O, Eliakim A. Relationship among repeated sprint tests, aerobic fitness, and anaerobic fitness in elite adolescent soccer players. J Strength Cond Res, 2009; 23(1): 163-169

Nassis GP, Geladas ND, Soldatos Y, Sotiropoulos A, Bekris V, Souglis A. Relationship between the 20-m multistage shuttle run test and 2 soccer-specific field tests for the assessment of aerobic fitness in adult semi-professional soccer players. J Strength Cond Res, 2010; 24(10): 2693-2697

Nesser TW, Latin RW, Berg K, Prentice E. Physiological determinants of 40-meter sprint performance in young male athletes. J Strength Cond Res, 1996; 10: 263-267

Osgnach C, Poser S, Bernardini R, Rinaldo R, Di Prampero PE. Energy cost and metabolic power in elite 
soccer: a new match analysis approach. Med Sci Sports Exerc, 2010; 42(1): 170-178

Rampinini E, Bishop D, Marcora SM, Bravo DF, Sassi R, Impellizzeri FM. Validity of simple field tests as indicators of match-related physical performance in top-level professional soccer players. Int J Sports Med, 2007; 28(03): 228-235

Ramsbottom R, Brewer J, Williams C. A progressive shuttle run test to estimate maximal oxygen uptake. $\mathrm{Br} \mathrm{J}$ Sports Med, 1988; 22: 141-144

Reilly T, Bangsbo J, Franks A. Anthropometric and physiological predispositions for elite soccer. J Sports Sci, 2000; 18(9): 669-683

Requena B, González-Badillo JJ, de Villareal ESS, Ereline J, García I, Gapeyeva H, Pääsuke M. Functional performance, maximal strength, and power characteristics in isometric and dynamic actions of lower extremities in soccer players. J Strength Cond Res, 2009; 23(5): 1391-1401

Stolen T, Chamari K, Castagna C, Wisloff, U. Physiology of soccer: An update. Sports Med, 2005; 35: 501-536

Strudwick T, Reilly T. Work-rate Profiles of Elite Premier League Football Players. Insight FA Coaches Assoc J, 2001; 4(2): 55-59

Tomlin DL, Wenger HA. The relationship between aerobic fitness and recovery from high intensity intermittent exercise. Sports Med, 2001; 31: 1-11

Wadley G, Le Rossignol P. The relationship between repeated sprint ability and the aerobic and anaerobic energy systems. J Sci Med Sport, 1998; 1: 100-110

Wisloff U, Castagna C, Helgerud J, Jones R, Hoff J. Strong correlation of maximal squat strength with sprint performance and vertical jump height in elite soccer players. Br J Sports Med, 2004; 38: 285-288

Wong PL, Chamari K, Dellal A, Wisløff U. Relationship between anthropometric and physiological characteristics in youth soccer players. J Strength Cond Res, 2009; 23(4): 1204-1210

Young W, Benton D, Duthie G, Pryor J. Resistance training for short sprints and maximum-speed sprints. Strength Cond J, 2001; 232: 7-13

Zając A, Jarząbek R, Waśkiewicz Z. The Diagnostic Value of the 10- and 30-Second Wingate Test for Competitive Athletes. J Strength Cond Res, 1999; 13(1): 16-19

Ziogas GG, Patras KN, Stergiou N, Georgoulis AD. Velocity at lactate threshold and running economy must also be considered along with maximal oxygen uptake when testing elite soccer players during preseason. J Strength Cond Res, 2011; 25(2): 414-419

\section{Corresponding author:}

\section{Michał Boraczyński (Ph.D.)}

Department of Public Health, Faculty of Health Sciences, University of Warmia and Mazury, 14C Żołnierska St., PL-10719 Olsztyn, Poland,

Phone: (+48) 533-101-720,

E-mail: michal.boraczynski@gmail.com 